\title{
Moving Target Detection and Tracking using Passive Acoustic Radar
}

\author{
Felix J. Yanovsky, Rustem B. Sinitsyn and Yevhen \\ Chervoniak \\ Electronics Department \\ National Aviation University \\ Kiev, Ukraine \\ yanovsky@nau.edu.ua; rsinitsyn@nau.edu.ua
}

\author{
Vitaliy Makarenko, Vadim Tokarev and Oleksandr \\ Zaporozhets \\ Department of Safety of Human Activities \\ National Aviation University \\ Kiev, Ukraine \\ zap@nau.edu.ua
}

\begin{abstract}
The moving target detection and tracking method for passive radars is investigated. The original algorithm, which is based on the wideband ambiguity function calculation, is suggested. The effectiveness of the suggested algorithm is proved by experimental measurements.
\end{abstract}

Keywords-acoustic signal, passive radar, difference of arrival ambiguity function, correlation function

\section{INTRODUCTION}

The passive methods of signal detection are very popular nowadays because of their effectiveness together with comparatively low cost of implementation. These methods are connected with use of self-radiation of the target in the radio frequency band or the sound, which is produced by the object, which is generating acoustic waves. No signal transmitter in this situation is required.

These methods can be used in different fields of human activity, for example, for controlling robots, measurements of different noises, generated by airplanes, cars, railway transport, for the task of environmental protection.

Recently, different self-guided vehicles and robotic aircrafts are becoming very popular. An automatic taxi has appeared and is operating now. Automatic helicopters (multicopters) are used to deliver goods to the consumer at home. Nevertheless, at the same time, these useful devices are increasing danger to surrounding people. Such a multicopter designed in the student design room of the electronics department at NAU is shown in fig. 1.

That is why the problem of automatic detection and tracking of such objects is rather vital. The methods using the same mathematical apparatus and technical principles are applicable in radio astronomy. All radio telescopes can be considered to be passive radars. Usually signals of passive radars are unknown and can be regarded as random. That is why statistical signal processing must be used in this case.

At the same time the signals which are used in passive radars are wideband. Therefore, the methods, which are used in wideband radars are useful [19].

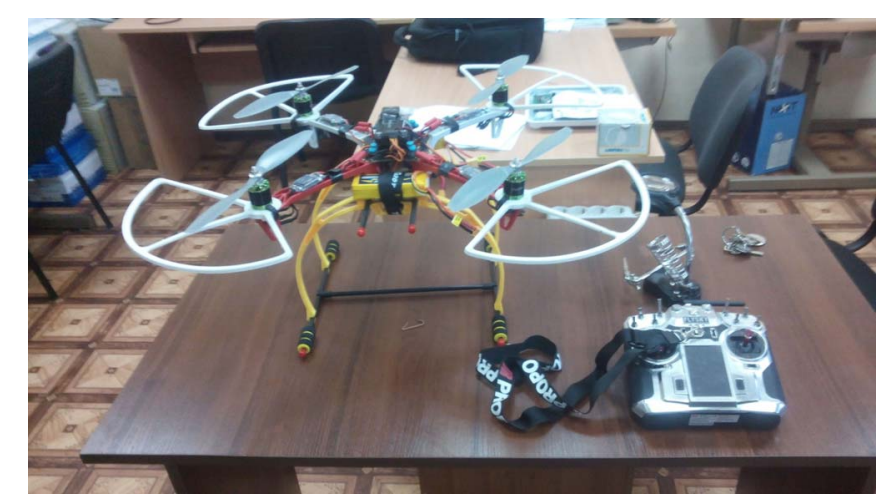

Fig. 1. Automatic multicopter designed in student design room of electronics department at the National Aviation University.

\section{Mathematical Principles}

\section{A. The Problem Statement}

The problem of the passive radar design in general is based on the inverse problem solution for radio waves or acoustic waves in case of an acoustic radar. Mathematical and engineering methods exist, for example [1,2], where such an approach is used.

But also different methods, which in mathematical sense can be considered as approximations of the correct mathematical solution, are also widely used. Among them one of the most popular is the method based on the difference of arrival $[3,4,5,6,7,8,9,10,17]$.

\section{B. Geometrical Describtion of the Signal Detection Problem}

The geometrical description of the signal detection problem depends on the field parameters we are using for this solution. We can use all parameters but very often for technical simplification, we use only a few parameters.

If only the time difference of arrival of waves at the antennas is used, the possible position of the object is limited by the locus of points, which are equidistant from antennas [6]. This locus is formed by the intersection of hyperboloids of revolution, which are formed by each pair of antennas 


$$
\begin{aligned}
& \sqrt{(x-x 1)^{2}+(y-y 1)^{2}+(z-z 1)^{2}}- \\
& \sqrt{(x-x 2)^{2}+(y-y 2)^{2}+(z-z 2)^{2}}=\text { const }
\end{aligned}
$$

where $(x 1, y 1, z 1)$ are the coordinates of the first antenna (microphone),

$(x 2, y 2, z 2)$ are the coordinates of the second antenna (microphone),

$(x, y, z)$ is the supposed position of the target.

The intersection of hyperboloids forming the area of possible target position is shown in fig. 2 .

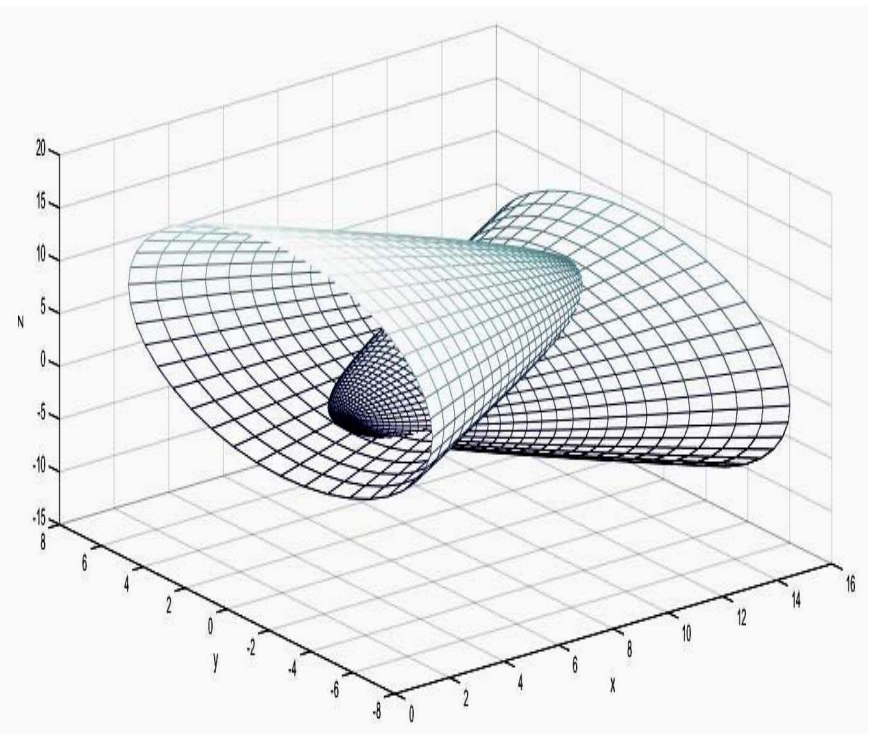

Fig. 2. Intesection of hyberboloids.

\section{Difference of Arrival Estimation}

The difference of arrival problem can be formulated as a problem of estimating the time shift between random processes, which are models of the received signals. For the digital sampled signal, this problem can be reduced to the problem of testing the statistical hypothesis about the form of the multivariate probability density function.

It can be proved, that after some assumptions, this problem can be reduced to the calculation of the correlation function. In order to obtain the estimate not depending on the possible velocity of the detected object the wideband ambiguity function is used instead of the correlation function [18].

$$
\begin{aligned}
& \chi(\mathbf{g}, \boldsymbol{\alpha})=\frac{1}{m \cdot n} \sum_{k=1}^{m} \sum_{j=1}^{n} \frac{\sqrt{\left|\alpha_{k j}\right|}}{N-g_{k j}} \times \\
& \sum_{i=g_{k j}+1}^{N}\left(\left(x_{k}(i \Delta t)\right)-m_{x_{k}}\right)\left(\left(y_{j}^{*}\left(\alpha_{k j}\left(i-g_{k j}\right) \Delta t\right)\right)-m_{y_{k}}\right)
\end{aligned},
$$

where $x$ and $y$ are signals in two antennas (microphones), $\alpha=\frac{c-v}{c+v}$ is a time-scale factor, which depends on the velocity of the target $v$ and the speed of sound (or electromagnetic wave) $c, m$ and $n$ are numbers of antennas (microphones), $N$ is the sample size.

The coefficient $\alpha$, which corresponds to the maximum of the ambiguity function for definite value $\tau$ is defined as

$$
\alpha^{*}(\tau)=\underset{\alpha}{\operatorname{argmax}}(\chi(\tau, \alpha)) .
$$

As a final result we receive a spatial (time) ambiguity function that does not depend on $\alpha$ and thus on the speed of movement of the target

$$
\chi_{S}(\tau)=\chi\left(\tau, \alpha^{*}(\tau)\right)
$$

The final signal detection is done by thresholding of the maximum of the ambiguity function value with the decision threshold. For stabilizing the false alarm probability the nonparametric variants of the ambiguity function were suggested $[12,13,14,15,16,20]$.

Final target tracking is done by direct calculation of the ambiguity function value in all points of the observed area. This allows us to solve the problem in the case of multiple targets.

\section{EXPERIMENTAL RESEARCH}

The record of the sound produced by the multicopter during the flight is presented in fig.3.

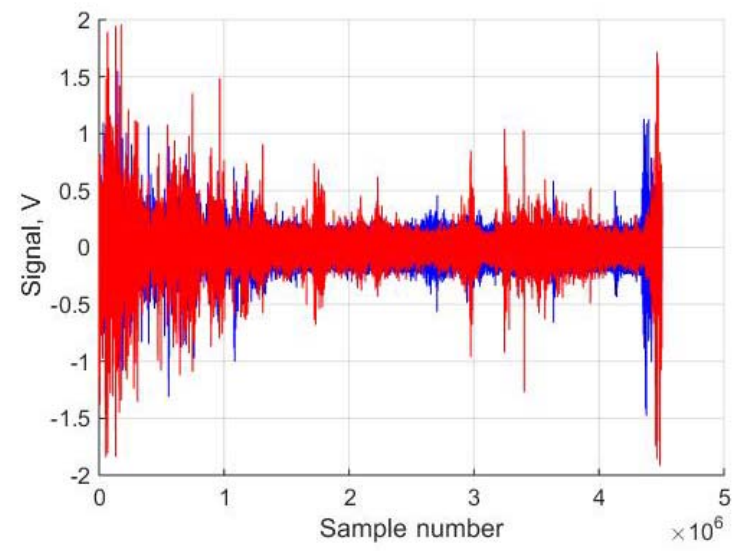

Fig. 3. Multicopter sound record.

Each 10000 sample units are forming a time window in which the ambiguity function is calculated with the help of the Fast Fourier Transform (FFT) algorithm for the two microphone signals, which are shown in fig. 4. For the first signal time is presented in time samples of ADC and for the second signal it is recalculated into the corresponding distance. 
The spectral densities of these signals are presented in fig.5. The calculated ambiguity function is demonstrated in fig. 6 and in fig 7 the spatial ambiguity function, which does not depend on the target velocity, is presented. In our experimental measurements we have used the signals from 3 microphones, using them as 2 pairs. Using the geometrical coordinates of the positions of microphones the projections of the spatial ambiguity functions to the horizontal plane were calculated for each pair of microphones (in fig.8 and fig.9) and as well the sum of the projections (in fig. 10).
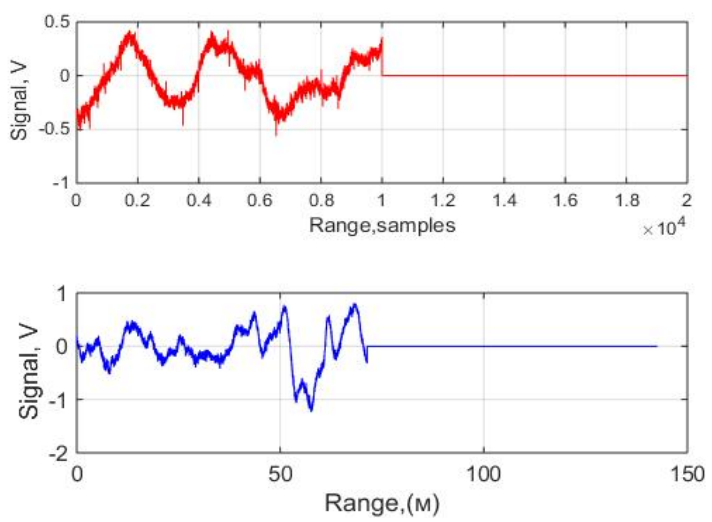

Fig. 4. The sound records for two microphones.
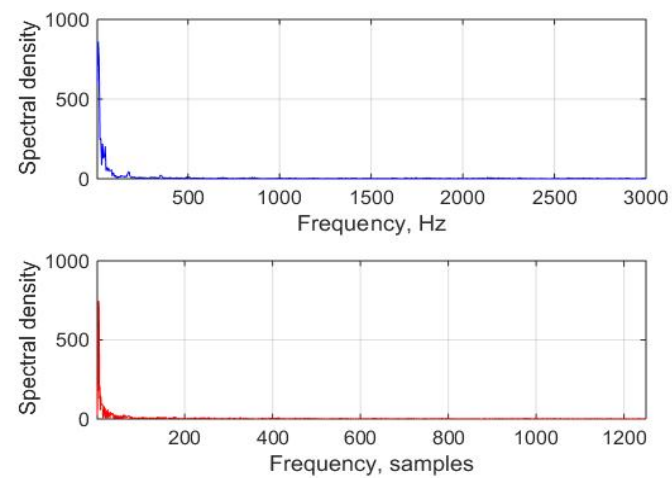

Fig. 5. Spectral density of signals for two microphones.

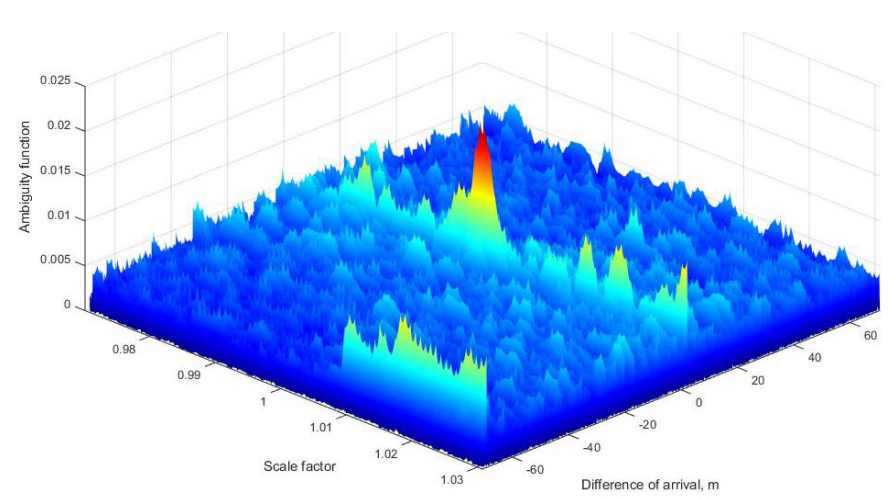

Fig. 6. Wideband ambiguity functioin of the signal of the multicopter in free space.

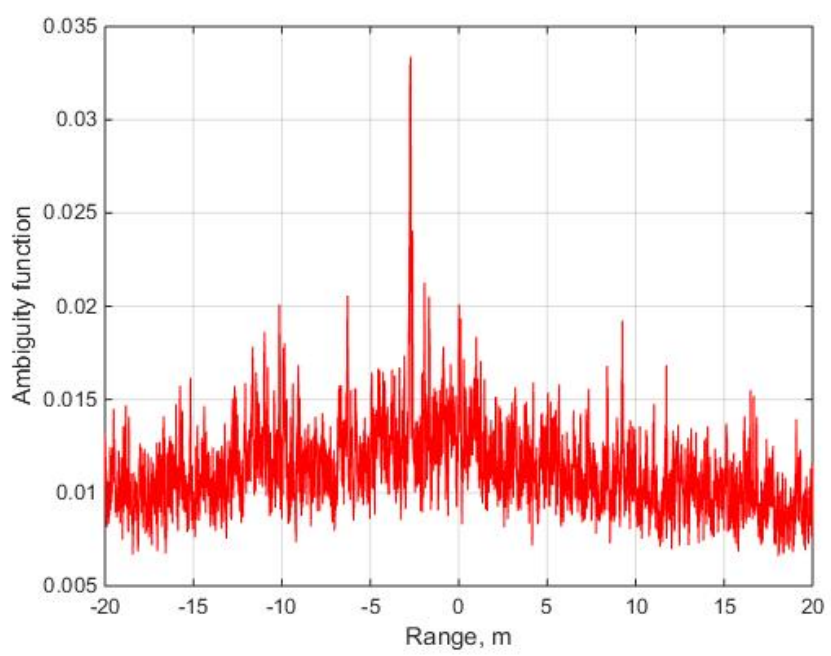

Fig. 7. Dependence of the maximum of the ambiguity function on distance.

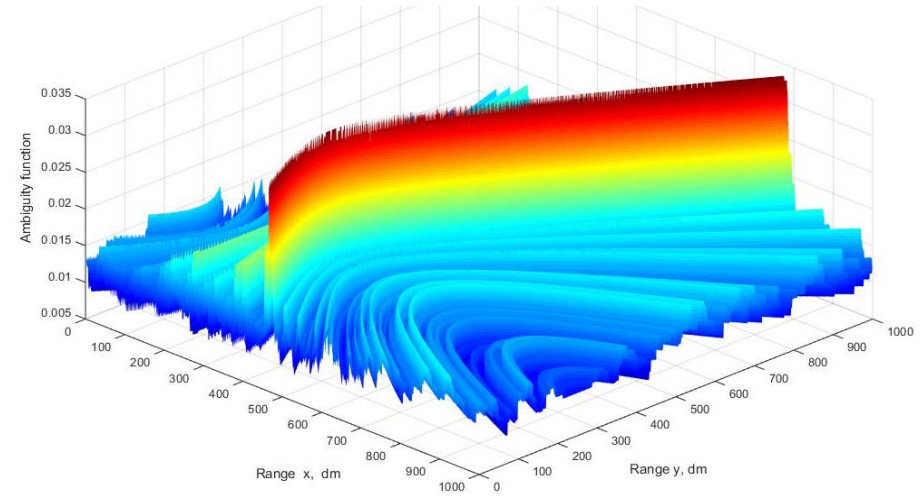

Fig. 8. Projection of the ambiguity function to the horizontal plane for the first pair of microphones.

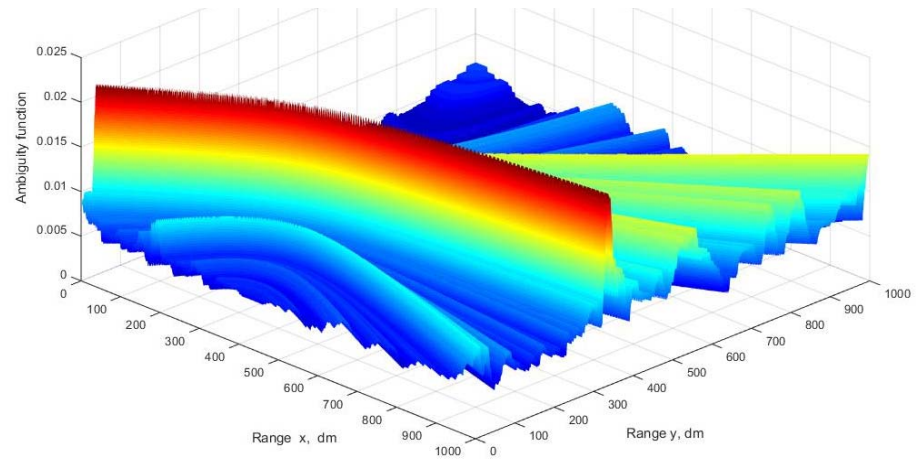

Fig. 9. Projection of the ambiguity function to the horizontal plane for the second pair of microphones. 


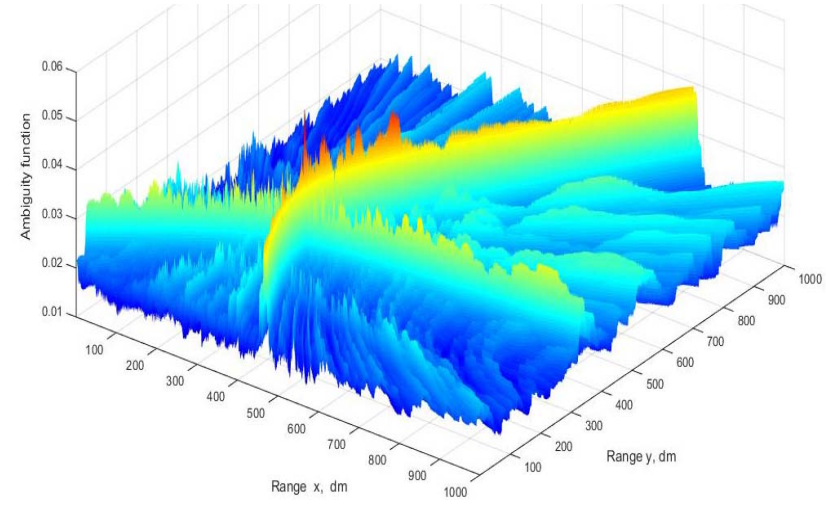

Fig. 10. The sum of projections of the ambiguity function to the horizontal plane for two pairs of microphones .

After thresholding, we obtain the detected objects and their coordinates. The additional information about the trajectory can be obtained on the basis of analyses of changing the estimated coordinates in time.

\section{CONCLUSIONS}

In the paper one of the methods of signal detection and tracking for passive acoustic radars has been developed. The use of the ambiguity function allowed obtaining the algorithm, which does not depend on the moving target velocity.

The suggested algorithm was tested by practical measurements and enables us to measure the coordinates of the flying multicopter. band.

The suggested technique can be used in the radio frequency

\section{ACKNOWLEDGMENT}

The authors are grateful to Denys Navrotskyi for the photo of the multicopter designed at the student design room of the electronics department at the National Aviation University under his supervision.

This work is supported by the Ministry of Education and Science of Ukraine in the framework of the project \# 1053DB16.

\section{REFERENCES}

[1] O. O. Drobakhin, G. G. Sherstyuk, A.G. Mamedov, and A.Yu.Tuluk, "Inverse Aperture Synthesis in the Intermediate Zone of Radiation for Radio Image Forming by Optimal Filtration with Evolutionary Control," Proceedings 2016 8th International Conference on Ultrawideband and Ultrashort Impulse Signals (UWBUSIS 2016), Odessa, Ukraine, 2016, pp. 109-111.

[2] G.A. Tyulepberdinova and S.A. Adilzhanova, "The inverse problem of acoustics and its reduction to the operator form" (Obratnaya zadacha akustiki I ee svedeniye $\mathrm{k}$ operatornomu vidu, International Journal of Applied and Fundamental Research, 2015, No 2-1, pp.30-32 (in Russian).

[3] J. C. Chen, L. Yip, J. Elson, Hanbiao Wang, D. Maniezzo, R.E. Hudson, Kung Yao, and D. Estrin, "Coherent acoustic array processing and localization on wireless sensor networks," Proceedings of the IEEE, Vol. 91, No. 8, August 2003, pp. 1154 - 1162.
[4] J. Lanslots, F. Deblauwe, and K. Janssens, "Selecting Sound Source Localization Techniques for Industrial Applications Sound and Vibration," Sound \& Vibration, June 2010, Leuven, Belgium, pp. 6 - 9.

[5] J.M. Valin, F. Michaud, and J. Rouat, Robust localization and tracking of simultaneous moving sound sources using beamforming and particle filtering, Robotics and Autonomous Systems, 2007, No 55(3), pp. 216228.

[6] F. Yanovsky and R. Sinitsyn, "Application of Wideband Signals for Acoustic Localization,” Proceedings 2016 8th International Conference on Ultrawideband and Ultrashort Impulse Signals (UWBUSIS 2016), Odessa, Ukraine, 2016, pp. 27-35.

[7] R. Sinitsyn, F. Yanovsky, E. Chervoniak, and O. Zaporozhets, "Determination of aircraft current location on the basis of its acoustic noise," Telecommunications and Radio Engineering, 2015, Vol. 74 (5), pp. 397-408.

[8] E. Chervoniak, R. Sinitsyn, and F. Yanovsky, "Retrieving aircraft motion parameters, using acoustic passive radar," Proceedings of the Signal Processing Symposium (SPSympo-2015), June 10-12, 2015, Debe, Poland, 2015, pp. 69-73.

[9] Ye. Chervoniak, R. Sinitsyn, and F. Yanovsky, "Signal detection algorithm for aircraft localization by means of passive acoustic radar," Proceedings of International Young Scientists Forum on Applied Physics (YSF-2015), Sept. 29-Oct. 2, 2015, Dnipropetrovsk, Ukraine, 2015, pp.1-4, DOI: 10.1109/YSF.2015.7333151.

[10] R. Sinitsyn, F. Yanovsky, E. Chervoniak, and O. Zaporozhets, "Passive acoustic radar for aircraft trajectory tracking," Proceedings of IEEE Microwaves, Radar and Remote Sensing Symposium (MRRS-2014), September 23-25, 2014, Kiev, Ukraine, 2014, pp.70-83.

[11] R. Sinitsyn and F. Yanovsky, "Wideband Copula Ambiguity Function in Radar and Navigation Systems," Electronics and Control Systems, 2014, No 4 (42), pp.11-17.

[12] R. Sinitsyn, F. Yanovsky, "Acoustic Noise Atmospheric Radar with Nonparametric Copula Based Signal Processing," Telecommunications and Radio Engineering, 2012, Vol. 71 (4), pp. 327-335.

[13] R. Sinitsyn and F. Yanovsky, "MIMO Radar Copula Ambiguity Function," Proceedings of the 9th European Radar Conference, Oct. 29 Nov. 2, 2012, Amsterdam, Netherlands, 2012, pp. 146-149.

[14] R. Sinitsyn and F. Yanovsky, "Non-Parametric Signal Processing in Noise Radar," Proceedings of Noise Radar Technology conference, September 27-29, 2012, Yalta, Ukraine, 2012, pp. 1-8.

[15] Zh. Bokal, R. Sinitsyn, and F. Yanovsky, Generalized Copula Ambiguity Function Application for Radar Signal Processing, Proceedings of Microwaves, Radar and Remote Sensing Symposium (MRRS), August 25 - 27, 2011, Kiev, Ukraine, 2011, pp. 313 - 316.

[16] R. Sinitsyn and F. Yanovsky, "Copula Ambiguity Function for Wideband Random Radar Signals," Proceedings of The International IEEE Conference on Microwaves, Communications Antennas and Electronic Systems (COMCAS), Nov 7-9, 2011, Tel-Aviv, Israel, 2011, pp. 1-4.

[17] W. Kam and B. Ferguson, "Methods for Computing the Passive Wideband Cross-Ambiguity Function," Proceedings Fifth International Congress on Sound and Vibration, December 15-18, 1997, Adelaide, South Australia, 1997, Vol. 4(2213), pp. 131-138.

[18] F. Yanovsky and R. Sinitsyn, "Ultrawideband signal processing algorithms for radars and sodars," Proceedings 2006 3rd International Conference on Ultrawideband and Ultrashort Impulse Signals (UWBUSIS 2006), Sevastopol, Ukraine, 2006, pp. 66-71.

[19] F.J. Yanovsky, V.E. Ivashchuk, and V.P. Prokhorenko, "Through-thewall surveillance te chnologies," Proceedings 2012 6th International Conference on Ultrawideband and Ultrashort Impulse Signals, (UWBUSIS 2012), Sevastopol, Ukraine, 2006, pp. 30-33.

[20] R. Sinitsyn and F. Yanovsky, "Kernel estimates of the characteristic function for radar signal detection", Proceedings 2nd European Radar Conference (EuRAD 2005), EuMA, IEEE, 2005, Paris, pp. 53-56. 